Selected Topics on Continuous-Time Controlled Markov Chains and Markov Games 
Series Editor: Dennis Barden (Univ. of Cambridge, UK)

\author{
Published \\ Vol. 1 Recent Progress in Conformal Geometry \\ by Abbas Bahri \& Yong Zhong Xu \\ Vol. 2 The Geometry of Curvature Homogeneous \\ Pseudo-Riemannian Manifolds \\ by Peter B. Gilkey \\ Vol. 3 Dynamics and Symmetry \\ by Michael J. Field \\ Vol. 4 The Solution of the $k(G V)$ Problem \\ by Peter Schmid \\ Vol. 5 Selected Topics on Continuous-Time Controlled \\ Markov Chains and Markov Games \\ by Tomás Prieto-Rumeau \& Onésimo Hernández-Lerma
}


ICP Advanced Texts in Mathematics - Vol. 5

\section{Selected Topics on Continuous-Time Controlled Markov Chains and Markov Games}

\section{Tomás Prieto-Rumeau}

Universidad Nacional de Educación a Distancia, Spain

Onésimo Hernández-Lerma CINVESTAV-IPN, Mexico 
Published by

Imperial College Press

57 Shelton Street

Covent Garden

London WC2H 9HE

Distributed by

World Scientific Publishing Co. Pte. Ltd.

5 Toh Tuck Link, Singapore 596224

USA office: 27 Warren Street, Suite 401-402, Hackensack, NJ 07601

UK office: 57 Shelton Street, Covent Garden, London WC2H 9HE

\section{British Library Cataloguing-in-Publication Data}

A catalogue record for this book is available from the British Library.

\section{ICP Advanced Texts in Mathematics - Vol. 5 \\ SELECTED TOPICS ON CONTINUOUS-TIME CONTROLLED MARKOV CHAINS AND MARKOV GAMES}

Copyright () 2012 by Imperial College Press

All rights reserved. This book, or parts thereof, may not be reproduced in any form or by any means, electronic or mechanical, including photocopying, recording or any information storage and retrieval system now known or to be invented, without written permission from the Publisher.

For photocopying of material in this volume, please pay a copying fee through the Copyright Clearance Center, Inc., 222 Rosewood Drive, Danvers, MA 01923, USA. In this case permission to photocopy is not required from the publisher.

ISBN-13 978-1-84816-848-0

ISBN-10 1-84816-848-9

Typeset by Stallion Press

Email: enquiries@stallionpress.com

Printed in Singapore. 
To Guadalupe and my parents, Emilio and Michèle.

To Marina, Gerardo, Adrián, Claudia, and Lucas. 
This page intentionally left blank 


\section{Preface}

This book concerns continuous-time controlled Markov chains and Markov games. The former, which are also known as continuous-time Markov decision processes, form a class of stochastic control problems in which a single decision-maker wishes to optimize a given objective function. In contrast, in a Markov game there are two or more decision-makers (or players, or controllers) each one trying to optimize his/her own objective function.

The main features of the control and game models studied in the book are that the time variable is continuous, the state space is denumerable, and the control (or action) sets are Borel spaces. Moreover, the transition and reward rates of the dynamical system may be unbounded. Controlled Markov chains and Markov games have many important applications in areas such as telecommunication networks, population and epidemic models, engineering, operations research, etc. Some of these applications are illustrated in this book.

We note that most of the material presented here is quite recent: it has been published in the last six years, and it appears in book form for the first time.

One of the main goals of this book is to study the so-called advanced optimality criteria for controlled Markov chains (e.g., bias, overtaking, sensitive discount, and Blackwell optimality), which are refinements of the basic criteria, namely, discounted and average reward optimality. To make this a self-contained book, we also give the main results on the existence of controlled Markov chains and the basic optimality criteria. For the corresponding technical details - some of which have been skipped here - the reader can consult Guo and Hernández-Lerma's Continuous-Time Markov Decision Processes: Theory and Applications [52].

A particular emphasis is made regarding the application of the results 
presented in the book. One of our main concerns is to propose assumptions on the control and game models that are easily verifiable (and verified) in practice. Furthermore, we study an algorithm to solve a certain class of control models, and establish some approximation results that allow us to give precise numerical approximations of the solutions to some problems of practical interest.

Hence, the book has an adequate balance between, on the one hand, theoretical results and, on the other hand, applications and computational issues. It is worth mentioning that the latter were, somehow, missing in the literature on continuous-time controlled Markov chains.

Finally, the topic of zero-sum two-person continuous-time Markov games, for both the basic - discounted and average payoff - and some "advanced" optimality criteria — bias and overtaking equilibria — appears for the first time in book form.

This book is mainly addressed to researchers in the fields of stochastic control and stochastic games. Indeed, it provides an extensive, rigorous, and up-to-date analysis of continuous-time controlled Markov chains and Markov games. It is also addressed to advanced undergraduate and beginning graduate students because the reader is not supposed to have a high mathematical background. In fact, a working knowledge of calculus, linear algebra, probability, and continuous-time Markov chains (at the level of, say, Chapter 4 of R. Durrett's book Essentials of Stochastic Processes [31]) should suffice to understand the material herein. As already mentioned, the reader interested in the theoretical foundations of controlled Markov chains can consult [52].

We have carefully written this book, with great dedication and commitment. We apologize, however, for any errors and omissions it might contain. 


\section{Contents}

Preface vii

1. Introduction 1

1.1 Preliminary examples . . . . . . . . . . . . . . . 1

1.1.1 A controlled population system . . . . . . . . . . 1

1.1.2 A prey-predator game model . . . . . . . . . 6

1.2 Overview of the book . . . . . . . . . . . . . . 9

1.3 Contents ............................ 12

1.4 Notation . . . . . . . . . . . . . . . . 13

2. Controlled Markov Chains $\quad 15$

2.1 Introduction . . . . . . . . . . . . . . . . . 15

2.2 The control model . . . . . . . . . . . . . . . 16

2.3 Existence of controlled Markov chains . . . . . . . . . . 19

2.4 Exponential ergodicity . . . . . . . . . . . . . . . . . . . 24

2.5 Proof of Theorem $2.11 \ldots \ldots \ldots$

2.6 Conclusions . . . . . . . . . . . . . . 40

3. Basic Optimality Criteria 43

3.1 Introduction . . . . . . . . . . . . . . . . 43

3.2 The finite horizon case . . . . . . . . . . . 46

3.3 The infinite horizon discounted reward . . . . . . . . . 49

3.3.1 Definitions . . . . . . . . . . . . . 49

3.3.2 The discounted reward optimality equation . . . 50

3.3.3 The uniformization technique . . . . . . . 56

3.3.4 A continuity theorem for discounted rewards . . 57 
3.4 The long-run expected average reward . . . . . . . . 59

3.5 The vanishing discount approach to average

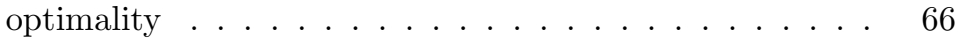

3.6 Pathwise average optimality . . . . . . . . . . 72

3.7 Canonical triplets and finite horizon control problems . . . . . . . . . . . . . 75

3.8 Conclusions . . . . . . . . . . . . . . . . . . 77

4. Policy Iteration and Approximation Theorems 81

4.1 Introduction . . . . . . . . . . . . . . . . . . . 81

4.2 The policy iteration algorithm . . . . . . . . . 83

4.2.1 Discounted reward problems . . . . . . . . 83

4.2.2 Average reward problems . . . . . . . . 86

4.3 Approximating discounted reward CMCs . . . . . . . . . 89

4.4 Approximating average reward CMCs . . . . . . . . . . 98

4.5 Conclusions . . . . . . . . . . . . . . . . . 112

5. Overtaking, Bias, and Variance Optimality 115

5.1 Introduction . . . . . . . . . . . . . . . . 115

5.2 Bias and overtaking optimality . . . . . . . . . 117

5.3 Variance minimization . . . . . . . . . . . . . 120

5.4 Comparison of variance and overtaking optimality . . . 126

5.5 Conclusions . . . . . . . . . . . . . . . . 128

6. Sensitive Discount Optimality 129

6.1 Introduction . . . . . . . . . . . . . . . . . 129

6.2 The Laurent series expansion . . . . . . . . . . . . . . 131

6.3 The vanishing discount approach (revisited) . . . . . 137

6.4 The average reward optimality equations . . . . . . . . 140

6.5 Strong discount optimality . . . . . . . . . . . . . 143

6.6 Sensitive discount optimality in the class of stationary policies . . . . . . . . . . . . . 147

6.7 Conclusions . . . . . . . . . . . . . . . . . 149

7. Blackwell Optimality 151

7.1 Introduction . . . . . . . . . . . . . . . 151

7.2 Blackwell optimality in the class of stationary policies . . 153

7.3 Blackwell optimality in the class of all policies . . . . . 155

7.4 Conclusions . . . . . . . . . . . . . . 163 
8. Constrained Controlled Markov Chains 165

8.1 Introduction . . . . . . . . . . . . . . . . 165

8.2 Discounted reward constrained CMCs . . . . . . . . . 167

8.3 Average reward constrained CMCs . . . . . . . . . 173

8.4 Pathwise constrained CMCs . . . . . . . . . . . 185

8.5 The vanishing discount approach to constrained CMCs . . . . . . . . . . . . . 187

8.6 Conclusions . . . . . . . . . . . . . . . 191

9. Applications 193

9.1 Introduction . . . . . . . . . . . . . . . . . . 193

9.2 Controlled queueing systems . . . . . . . . . . . . 196

9.3 A controlled birth-and-death process . . . . . . . . 199

9.4 A population system with catastrophes . . . . . . . . 209

9.5 Controlled epidemic processes . . . . . . . . . . . . 219

9.6 Conclusions . . . . . . . . . . . . . . . . 225

10. Zero-Sum Markov Games 227

10.1 Introduction . . . . . . . . . . . . . . . . 227

10.2 The zero-sum Markov game model . . . . . . . . . . 228

10.3 Discount optimality . . . . . . . . . . . . . . . . . 233

10.4 Average optimality . . . . . . . . . . . . 236

10.5 The family of average optimal strategies . . . . . . . . 242

10.6 Conclusions . . . . . . . . . . . . . . . . 244

11. Bias and Overtaking Equilibria for Markov Games 245

11.1 Introduction . . . . . . . . . . . . . . . . . 245

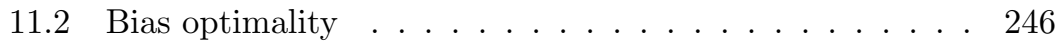

11.3 Overtaking optimality . . . . . . . . . . . . . 250

11.4 A counterexample on bias and overtaking optimality . . . . . . . . . . . . . 252

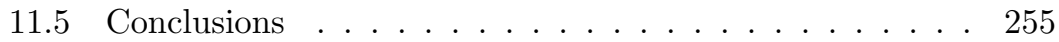

$\begin{array}{ll}\text { Notation List } & 257\end{array}$

$\begin{array}{ll}\text { Bibliography } & 265\end{array}$

Index 